\title{
2MASS observations of Wolf-Rayet stars and galaxies
}

\author{
Schuyler D. Van Dyk, Thomas H. Jarrett, and Roc M. Cutri \\ Infrared Processing and Analysis Center, Caltech, Pasadena, CA, USA \\ Michael F. Skrutskie \\ University of Massachusetts, Amherst, MA, USA
}

\begin{abstract}
The Two Micron All Sky Survey (2MASS) has observed a number of Wolf-Rayet stars and galaxies at $J(1.25 \mu \mathrm{m}), H(1.65 \mu \mathrm{m})$, and $K_{s}(2.17$ $\mu \mathrm{m})$, as part of its systematic coverage of the entire sky with automated $1.3-\mathrm{m}$ telescopes at Mt. Hopkins, AZ, and CTIO, Chile. The cameras observe the sky in the three channels simultaneously, using $256 \times 256 \mathrm{HgCdTe}$-detector arrays. The survey samples the sky in $6^{\circ} \times 8$ ! 3 tiles. The $2 M A S S$ Production Processing System transforms the raw binary survey data to final atlas images and source extractions with precise photometric calibration and astrometric positions. The survey's $10 \sigma$ sensitivity is $15.8 \mathrm{mag}$ at $J, 15.1$ at $H$, and 14.3 at $K_{s}$. In this poster we will present results from our analysis of several objects. We have observed WR stars both with and without associated ring nebulae. 2MASS imaging of the WR galaxies is far less affected by extinction, which can obscure much of the star formation activity. We will place these starburst dwarf galaxies in context with other galaxies, based on their near-IR colors and properties.
\end{abstract}

\section{Introduction}

56 Wolf-Rayet stars (van der Hucht et al. 1981) and 10 galaxies (Conti 1991) have been observed as part of routine nightly northern and southern operations. Data including ten WR stars and six galaxies had been processed by the 2MASS Production Pipeline. The WR stars include several with ring nebulae (Miller \& Chu 1993). Sample 2MASS Atlas Images of selected WR stars and galaxies can be found in the Image Gallery at http://www.ipac.caltech.edu/2mass/.

\section{Preliminary results}

All the WR stars, except WR 102, are detected by 2MASS. The magnitudes agree well with published values. WR star near-IR colors are affected by emission lines, free-free emission and thermal dust emission from mass-loss-produced circumstellar shells, and extinction. We detect the M1-67, NGC 2359, and Carina ring nebulae. None of the other ring nebulae are detectable in the $2 M A S S$ data. This implies that the $\mathrm{H}_{2}$ excitation mechanism is more likely to be shockexcitation of the ISM, rather than UV flourescence.

Four of the six galaxies are IRAS sources. III Zw 107 appears to be an interacting galaxy pair; both galaxies are compact and IR-bright. Mrk 309 is 

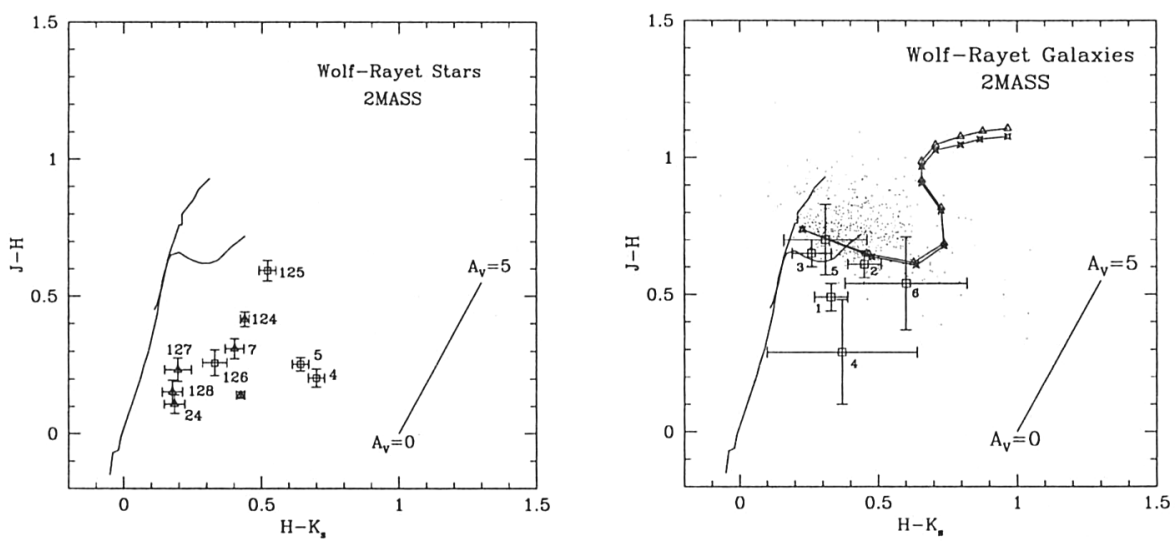

Figure 1. Color-color diagram for the WR stars and galaxies. The triangles are those WR stars with ring nebulae. For the galaxies: $1=\operatorname{III} \mathrm{Zw} 107$, $2=\operatorname{Mrk} 309,3=\operatorname{Mrk} 712,4=\operatorname{Mrk} 724,5=\operatorname{Mrk} 750$, and $6=\operatorname{Pox} 139$. The small points are galaxies from the 2MASS Sampler. $K$-correction tracks are for ellipticals (triangles) and spirals (diamonds); each point is $\Delta z=0.10$. Also shown on both diagrams are the stellar tracks for dwarfs and giants from Bessell \& Brett (1988). A reddening vector is shown, assuming the IR extinction law from Rieke \& Lebofsky (1985).

dominated by its bright Seyfert nucleus, but a very red component is seen at the northwest end of its disk. Mrk 712 is also dominated by its central component, and also has a very red knot to the northeast. Likewise for Mrk 750. The other two galaxies, Mrk 724 and Pox 139, are significantly bluer. Mrk 724 is a blue compact dwarf, whereas Pox 139 appears as a dwarf late-type spiral. The bluer galaxies, in particular, have colors consistent with individual WR stars.

\section{References}

Bessell, M.S., Brett, J.M. 1988, PASP 100, 1134

Conti, P.S. 1991, ApJ 377, 115

Miller, G.J., Chu, Y.-H. 1993, ApJS 85, 137

Rieke, G.H, Lebofsky, M.J. 1985, ApJ 288, 618

van der Hucht, K.A., Conti, P.S., Lundström, I., Stenholm, B. 1981, SSR 28227 